\title{
Highly Differentiated Projection-Specific Cortical Subnetworks
}

\author{
Mieko Morishima, ${ }^{1,2,3}$ Kenji Morita, ${ }^{4}$ Yoshiyuki Kubota, ${ }^{1,2,3}$ and Yasuo Kawaguchi ${ }^{1,2,3}$ \\ ${ }^{1}$ Division of Cerebral Circuitry, National Institute for Physiological Sciences, Graduate University for Advanced Studies (SOKENDAI), Okazaki, 444-8787, \\ Japan, ${ }^{2}$ Department of Physiological Sciences, Graduate University for Advanced Studies (SOKENDAI), Okazaki 444-8585, Japan, ${ }^{3}$ Japan Science and \\ Technology Agency, CREST (Core Research for Evolutional Science and Technology), Tokyo 102-0075, Japan, and ${ }^{4}$ Department of Cognitive Neuroscience, \\ Graduate School of Medicine, The University of Tokyo, Tokyo 113-0033, Japan
}

Pyramidal cells in the neocortex are differentiated into several subgroups based on their extracortical projection targets. However, little is known regarding the relative intracortical connectivity of pyramidal neurons specialized for these specific output channels. We used paired recordings and quantitative morphological analysis to reveal distinct synaptic transmission properties, connection patterns, and morphological differentiation correlated with heterogeneous thalamic input to two different groups of pyramidal cells residing in layer 5 (L5) of rat frontal cortex. Retrograde tracers were used to label two projection subtypes in L5: crossed-corticostriatal (CCS) cells projecting to both sides of the striatum, and corticopontine (CPn) cells projecting to the ipsilateral pons. Although CPn/CPn and CCS/CCS pairs had similar connection probabilities, $\mathrm{CPn} / \mathrm{CPn}$ pairs exhibited greater reciprocal connectivity, stronger unitary synaptic transmission, and more facilitation of paired-pulse responses. These synaptic characteristics were strongly correlated to the projection subtype of the presynaptic neuron. CPn and CCS cells were further differentiated according to their somatic position (L5a and L5b, the latter denser thalamic afferent fibers) and their dendritic/axonal arborizations. Together, our data demonstrate that the pyramidal projection system is segregated into different output channels according to subcortical target and thalamic input, and that information flow within and between these channels is selectively organized.

\section{Introduction}

A prominent feature of neocortical pyramidal cells is their diverse projections to various brain areas (Jones, 1984; Gabbott et al., 2005; Shipp, 2007). In addition to layer-specific extracortical projection patterns, it has recently become evident that individual cortical layers contain multiple pyramidal cell subtypes with different axonal targets (Molnár and Cheung, 2006) and distinct intracortical connections (Morishima and Kawaguchi, 2006; Le Bé et al., 2007; Brown and Hestrin, 2009; Sato and Svoboda, 2010). However, the details of intracortical connectivity between these projection subtypes of pyramidal cells remain to be examined.

The frontal cortex has notable functional linkages with the basal ganglia and cerebellum that are important for action and cognition (Ito, 1984; Wilson, 2004). Layer 5 (L5) pyramidal cells in frontal cortex project to the striatum (cortico-basal ganglia pathway) and pontine nuclei (a relay in the cortico-cerebellar

Received Feb. 12, 2011; revised May 16, 2011; accepted May 19, 2011.

Author contributions: M.M. and Y. Kawaguchi designed research; M.M. performed research; M.M., K.M., Y. Kubota, and Y. Kawaguchi analyzed data; M.M. and Y. Kawaguchi wrote the paper.

This work was supported by Japan Science and Technology Agency, CREST (Core Research for Evolutional Science and Technology), and Grant-in-Aid for Scientific Research (KAKENHI) 21240030 to Y.K.; and by KAKENHI 21700366 from Japan Ministry of Education, Culture, Sports, Science, and Technology and 20246026 from Japan Society for the Promotion of Science to K.M. We thank Sayuri Hatada for experimental help, Dr. Allan T. Gulledge for helpful discussions and suggestions, and Drs. Ariel Agmon and Fuyuki Karube for comments on the early version of this manuscript.

Correspondence should be addressed to Yasuo Kawaguchi, Division of Cerebral Circuitry, National Institute for Physiological Sciences, Okazaki, 444-8787, Japan. E-mail: yasuo@nips.ac.jp.

DOI:10.1523/JNEUROSCI.0772-11.2011

Copyright $\odot 2011$ the authors $\quad 0270-6474 / 11 / 3110380-12 \$ 15.00 / 0$ pathway). Output from the basal ganglia and cerebellum, in turn, feeds back to the frontal cortex via thalamic nuclei (Alexander et al., 1986; Jones, 2007; Ito, 2008; Strick et al., 2009). While interactions of these two loops within the cortex are essential for normal motor and cognitive functions (Middleton and Strick, 2000), the details of how neurons participating in these loops interact in the cortex remain unknown (Kawaguchi, 1997, 2003).

To better understand the cortical circuits responsible for output to the cerebellum and basal ganglia, we have identified crossed corticostriatal (CCS) cells projecting to the contralateral striatum (cortico-basal ganglia pathway) and corticopontine (CPn) cells that project to the pons (cortico-cerebellar pathway) (Wilson, 1987; Reiner et al., 2003; Morishima and Kawaguchi, 2006). These two subtypes form direction-selective synaptic connections from CCS to CPn cells (Morishima and Kawaguchi, 2006), suggesting a hierarchical organization among pyramidal cells based on their subcortical targets. Recently, differences have been reported in the connection probability between pyramidal cells projecting to specific subcortical targets, such as the tectum, striatum, or contralateral cortex (Brown and Hestrin, 2009). However, little is known about how cortical circuits are organized in relation to their subcortical outputs and inputs from subcortical regions. Here we compare synaptic transmission and intracortical connectivity in CPn/CPn and CCS/CCS pairs, and assess their dendritic/axonal distributions in relation to thalamic inputs. As a result, we report that individual projection-specific subnetworks are highly differentiated in connectional and temporal characteristics. 
A

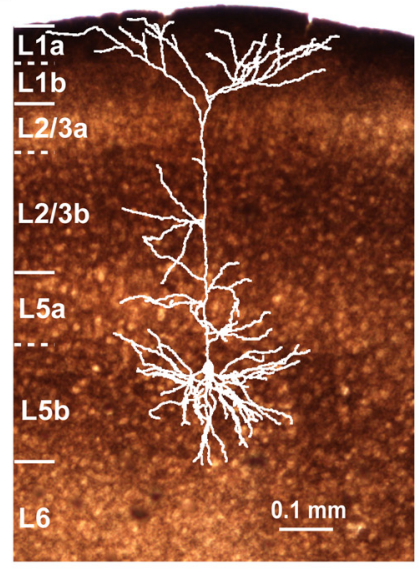

\section{C1 Basal dendrites} CPn CCS
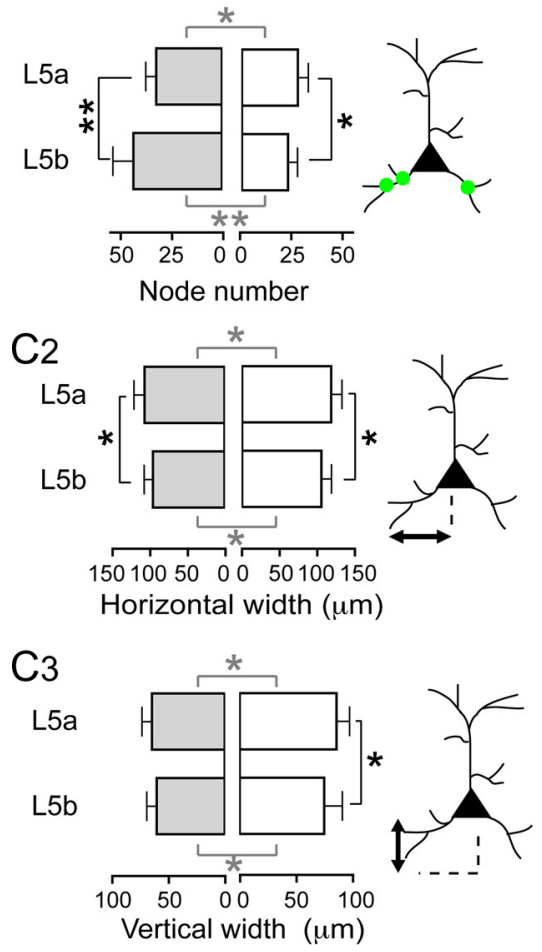

Figure 1. L5 Projection subtype-and sublamina-dependent dendritic branching. $A$, Sublaminar identification of the frontal cortex by VGLUT2-immunoreactive fiber distribution. L1a, L2/3b, and L5b, VGLUT2-rich regions (superficial, middle, and deep thalamic input zones). Borders between sublaminae (distance from pia; 8 sections), L1a:L1b border, $47 \pm 7 \mu \mathrm{m} ; \mathrm{L} 1 \mathrm{~b}: \mathrm{L} 2 / 3 \mathrm{a}, 122 \pm 13 \mu \mathrm{m} ; \mathrm{L} 2 / 3 \mathrm{a}: \mathrm{L} 2 / 3 \mathrm{~b}, 244 \pm$ $19 \mu \mathrm{m} ; \mathrm{L} 2 / 3 \mathrm{~b}: L 5 \mathrm{a}, 557 \pm 16 \mu \mathrm{m} ; \mathrm{L} 5 \mathrm{a}: \mathrm{L5} \mathrm{b}, 775 \pm 13 \mu \mathrm{m} . \boldsymbol{B}$, Dendrite reconstruction of CPn and CCS cells. L5 was divided into VGLUT2poor L5a and VGLUT2-rich L5b. $C_{1}-C_{3}$, Comparisons of three basal dendrite parameters: branch point (node) number, and horizontal and vertical width in L5a CPn $(n=12)$, L5b CPn $(n=22)$, L5a CCS $(n=12)$, and L5b CCS cells $(n=15)$. Note that basal dendrite morphology was differentiated depending on both subtype and sublamina. $D$, Comparison of summed tuft length in $\mathrm{L} 1$ of $\mathrm{L} 5 \mathrm{a} \mathrm{CPn}(n=9), \mathrm{L} b \mathrm{~b} C \mathrm{Pn}$ $(n=19)$, L5a CCS $(n=11)$, and L5b CCS cells $(n=14)$. The tuft length of CPn cells was longer than that of CCS cells. Tuft length of CCS cells was differentiated according to sublaminae in which their somata were located. $\boldsymbol{E}$, Comparison of summed dendritic length of oblique branches in L2/3 of L5a CPn $(n=11), \operatorname{Lb} \mathrm{b} C \mathrm{Pn}(n=13), \mathrm{L} 5 \mathrm{aCCS}(n=8)$, and L5bCCS cells $(n=12)$. Note that very few oblique branches were found in $L 5 b$ CPn cells. Error bar, S.D; ${ }^{*} p<0.05 ;{ }^{* *} p<0.01$ (gray, comparison between the subtypes of the same sublamina; black, comparison of the same subtype in different sublaminae).

\section{Materials and Methods}

Retrograde labeling of pyramidal cells. All experiments were performed in compliance with the guidelines for animal experimentation of the Okazaki National Research Institutes. Young male and female Wistar rats [postnatal day 17 (p17)-p21] were anesthetized with ketamine (40 mg/ $\mathrm{kg}$, i.m.) and xylazine ( $4 \mathrm{mg} / \mathrm{kg}$, i.m.). The following retrograde tracers were used: Alexa Fluor 555-conjugated cholera toxin subunit B (CTB555, Invitrogen), and red fluorescent latex microspheres (RetroBeads, Lumafluor). The tracers $(80-100 \mathrm{nl})$ were injected into the pons $(5.6 \mathrm{~mm}$ posterior to bregma, $0.8 \mathrm{~mm}$ lateral to bregma, depth $9-9.5 \mathrm{~mm}$ ) or contralateral striatum through glass pipettes by pressure application (PV820, WPI). In the case of striatal injection, the cortex, hippocampus, and fimbria just caudal to the striatum were removed by suction, and the tracer was applied obliquely through the lateral ventricle under direct observation to prevent tracer spilling into the cortex (Morishima and Kawaguchi, 2006).

Slice preparation. Two or $3 \mathrm{~d}$ after the tracer injection, oblique horizontal sections $300 \mu \mathrm{m}$ thick (p19-p23) were made from the medial agranular area of frontal cortex in an ice-cold buffered solution [containing (in $\mathrm{mM}$ ): 124 $\mathrm{NaCl}, 3 \mathrm{KCl}, 1 \mathrm{CaCl}_{2}, 5 \mathrm{MgCl}_{2}, 26 \mathrm{NaHCO}_{3}, 1$ $\mathrm{NaH}_{2} \mathrm{PO}_{4}, 10$ glucose, 1 lactic acid] equilibrated with a mixture of $95 \% \mathrm{O}_{2}$ and $5 \% \mathrm{CO}_{2}$. These slices were transferred to a holding chamber filled with a solution [containing (in $\mathrm{mm}$ ): $124 \mathrm{NaCl}, 3 \mathrm{KCl}, 1.2 \mathrm{CaCl}_{2}, 2.4 \mathrm{MgCl}_{2}, 26$ $\mathrm{NaHCO}_{3}, 1 \mathrm{NaH}_{2} \mathrm{PO}_{4}, 10$ glucose, 1 lactic acid] equilibrated with a mixture of $95 \% \mathrm{O}_{2}$ and $5 \%$ $\mathrm{CO}_{2}$. The holding chamber was maintained at $32^{\circ} \mathrm{C}$ for $20 \mathrm{~min}$ and then allowed to cool to room temperature. Lactic acid was omitted from the saline solution during experiments.

Electrophysiological recording. The pipette solution for whole-cell recording consisted of the following (in $\mathrm{mm}$ ): 130 potassium gluconate, $2 \mathrm{KCl}, 2 \mathrm{MgCl}_{2}$, 3 ATP, $0.3 \mathrm{GTP}, 10$ HEPES, and 20 biocytin, pH 7.0, 290 mOsm. Retrograde labeling cells were identified by fluorescence microscopy (BX50WI, Olympus) under a $40 \times$ immersion objective. Whole-cell recording was performed using the EPC 9 Double (HEKA Electronik). Recording temperature ranged from 29 to $31^{\circ} \mathrm{C}$. The depth of recorded neurons was $>50 \mu \mathrm{m}$ from the slice surface.

EPSC analysis. EPSCs were induced by single presynaptic action potentials generated by depolarizing somatic current pulses (duration, 5-6 ms) in the presynaptic cells and recorded in postsynaptic neurons voltage-clamped at $-60 \mathrm{mV}$. Signals were digitized at a $20 \mathrm{kHz}$ sampling rate. Series resistance was monitored periodically by delivery of small voltage pulses $(-5 \mathrm{mV}, 10 \mathrm{~ms})$. The series resistance of recording neurons, ranging from 6 to $25 \mathrm{M} \Omega$, was used for analysis. Electrophysiological data were analyzed by IGOR Pro (WaveMetrics). Input resistances were $62.2 \pm 18.11 \mathrm{M} \Omega$ in CPn cells $(n=30)$ and $111 \pm 40.9 \mathrm{M} \Omega$ in CCS cells $(n=28)$. Membrane time constants were $15.5 \pm 4.6 \mathrm{~ms}$ in CPn cells $(n=30)$ and $22.6 \pm$ $11.5 \mathrm{~ms}$ in CCS cells $(n=28)$. To analyze the EPSC characteristics, the peaks of presynaptic spikes were aligned and postsynaptic responses were averaged from at least 20 trials. To obtain the peak current of each trace, the current amplitudes (time window, 0.2 $\mathrm{ms}$ ) were averaged around the EPSC maximum. The baseline current was defined as the averaged current in a window (10 ms duration) before application of depolarizing current pulses to the presynaptic cell, which was selected based on the averaged traces. The peak EPSC is the peak current minus the baseline. The EPSC rise time was time from 20 to $80 \%$ 
Table 1. Unitary EPSC properties of CPn and CCS connections

\begin{tabular}{|c|c|c|c|}
\hline & $\mathrm{CPn} / \mathrm{CPn}$ & $\operatorname{cCS} / \operatorname{CSS}^{a}$ & $\mathrm{CCS} / \mathrm{CPn}^{b}$ \\
\hline Latency (ms) & $1.6 \pm 0.3(55)$ & $1.5 \pm 0.5(30)$ & $1.8 \pm 0.5(11)$ \\
\hline Rise time $(\mathrm{ms})^{c}$ & $1.2 \pm 0.45(54)$ & $1.0 \pm 0.4(29)$ & $1.1 \pm 0.5(11)$ \\
\hline Decay time constant (ms) & $6.9 \pm 1.9(49)$ & $6.8 \pm 1.9(29)$ & $6.3 \pm 2.5(11)$ \\
\hline \multicolumn{4}{|l|}{ Amplitude (pA) } \\
\hline Total connections & $\begin{array}{l}32.3 \pm 27.4^{* *}(63) \\
\text { (median, 22.8) }\end{array}$ & $\begin{array}{l}17.0 \pm 13.7(32) \\
\text { (median, 13.9) }\end{array}$ & $\begin{array}{l}14.7 \pm 9.2(11) \\
\text { (median, 11.1) }\end{array}$ \\
\hline L5a/L5a & $30.6 \pm 29.9(23)$ & $14.1 \pm 10.2(6)$ & $13.7 \pm 6.4(3)$ \\
\hline$L 5 b / L 5 b$ & $37.1 \pm 28.3(27)$ & $22.8 \pm 19.0(13)$ & $17.2 \pm 13.6(4)$ \\
\hline $\mathrm{L} 5 \mathrm{a} / \mathrm{L} 5 \mathrm{~b}$ & $23.0 \pm 18.5(4)$ & $14.6 \pm 3.3(4)$ & $15.6(1)$ \\
\hline CV of the amplitude & $0.59 \pm 0.28(63)$ & $0.53 \pm 0.21(32)$ & $0.50 \pm 0.12(11)$ \\
\hline Paired-pulse ratio & $1.27 \pm 0.34^{* *}(60)$ & $0.87 \pm 0.34(22)$ & $0.85 \pm 0.05(7)$ \\
\hline
\end{tabular}

Numbers in parentheses, number of pairs. Values are mean $\pm S D ;{ }^{* *} p<0.01$.

${ }^{a}$ Includes some data from the work of Morishima and Kawaguchi (2006) [latency, rise time, decay time constant, amplitude, CV of the amplitude, $n=24$; paired-pulse ratio, $n=15]$.

${ }^{b}$ Data taken from Morishima and Kawaguchi (2006)

'Time from 20\% to $80 \%$ of EPSC peak amplitude.

of the peak EPSC amplitude. The onset was defined as the point at which a line extrapolated from the rise time crossed the baseline. Latency was measured from the peak of presynaptic spike to the EPSC onset. The decay time constant was obtained by fitting a single exponential. The coefficient of variation (CV) of EPSC was corrected for baseline noise by subtracting the variance of the baseline noise from the variance of the EPSP peak amplitude. The baseline noise was obtained by selecting a second baseline region and amplitude window identical in durations to EPSC amplitude measurement and separated by the same interval (Feldmeyer et al., 1999). Trials showing failures of synaptic transmission were included when quantifying mean EPSC amplitude and paired-pulse responses.

To further characterize the dynamical property of the synaptic transmissions, amplitudes of EPSCs in response to a train of presynaptic spikes $(8$ spikes at $10 \mathrm{~Hz}$, at $0,100, \ldots, 700 \mathrm{~ms}$, and 1 spike at $500 \mathrm{~ms}$ later, i.e., at $1200 \mathrm{~ms}$; actual timings slightly vary across trials due to jitter of presynaptic spike time) were fitted to a mathematical model of shortterm synaptic plasticity (phenomenological approach) (Markram et al., 1998; Tsodyks and Markram, 1997; Wang et al., 2006). The model describes the dynamics of two variables, $x(0<x<1)$, which represents the normalized amount of available resources, and $u(0<u<1)$, which represents the fraction of resources used by each spike and can thus be considered to be analogous to the release probability. Upon the arrival of each spike, $x$ decreases by the amount of $u x$, while $u$ increases, presumably reflecting an increase of the residual calcium level, by the amount of $U(1-u)$, where $U$ is a constant that also represents the baseline level of $u$ in this model. Between spikes, $x$ and $u$ recover to their baseline levels ( $x=1, u=U$ ) with time constants $\tau_{\mathrm{D}}$ (recovery from depression) and $\tau_{\mathrm{F}}$ (recovery from facilitation), respectively. Collectively, the model is formulated as the following differential equation (Mongillo et al., 2008):

$$
\begin{aligned}
& d x / d t=(1-x) / \tau_{\mathrm{D}}-u x \delta\left(t-t_{\mathrm{sp}}\right) \\
& d u / d t=(U-u) / \tau_{\mathrm{F}}+U(1-u) \delta\left(t-t_{\mathrm{sp}}\right),
\end{aligned}
$$

where $\delta$ is Dirac delta function and $t_{\mathrm{sp}}$ represents time of presynaptic spike. The amplitude of postsynaptic conductance at each spike is proportional to the amount of resource used for the spike (i.e., $\mathrm{Cux}$, where $C$ is a scaling factor). Analytically solving this equation gives formulae of $u$, $x$, and, thereby, EPSC amplitude at each $t_{\mathrm{sp}}$, iteratively, as a function of the parameters $\left(U, \tau_{\mathrm{D}}, \tau_{\mathrm{F}}\right.$, and $\left.C\right)$ (cf. Markram et al., 1998; Tsodyks and Markram, 1997). A set of parameters that minimizes the sum of squares of the percentage difference between the predicted and the measured EPSC amplitudes was obtained by running an optimization function in MATLAB (MathWorks Inc.) with various initial values.

Histology. Slices containing biocytin-loaded cells were fixed in $4 \%$ paraformaldehyde, $1.25 \%$ glutaraldehyde, and $0.2 \%$ picric acid overnight at $4^{\circ} \mathrm{C}$, and resectioned at a thickness of $50 \mu \mathrm{m}$. The sections were incubated with avidin-biotin-peroxidase complex (1\%; ABC Elite, Vector Laboratories) in $0.05 \mathrm{M}$ Tris-buffered saline (TBS) containing $0.04 \%$
A
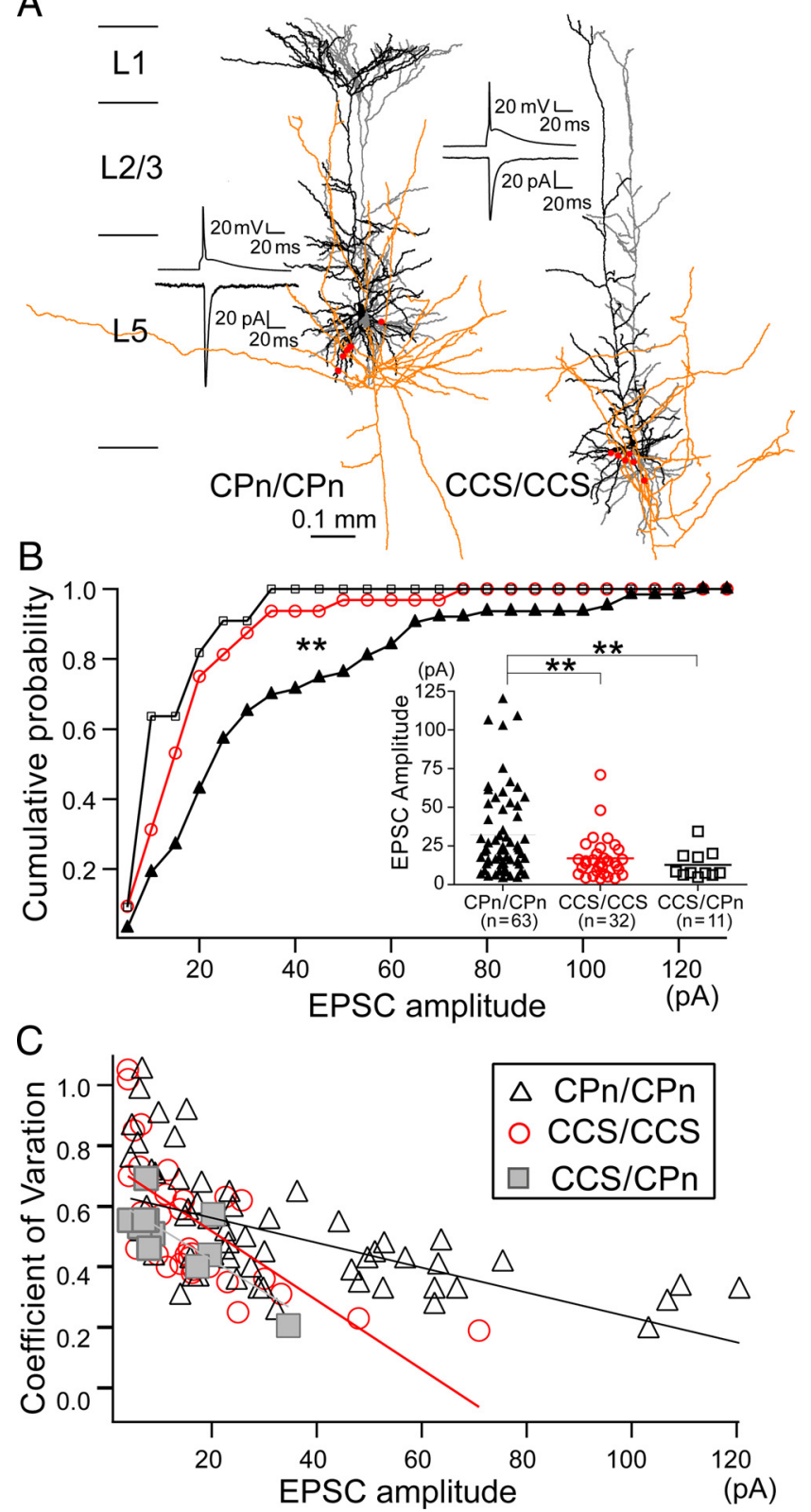

Figure 2. Different EPSC characteristics between $C P n / C P n$ and CCS/CCS connections. A, Synaptic connections of CPn/CPn and CCS/CCS. Insets, Presynaptic action potential and EPSC. Reconstruction of presynaptic dendrites (gray), axons (orange), and postsynaptic dendrites (black). Putative contact sites on postsynaptic dendrites are shown by red circles. B, Cumulative frequency distributions and scatter plots of EPSC amplitudes in three types of connections. Number of connections is shown in parentheses. In cumulative plot, ${ }^{* *} p<0.01$ (KolmogorovSmirnov test); in scatter plots, ${ }^{* *} p<0.01$ (ANOVA, post hoc Tukey's test). C, Relation between CV of EPSC amplitude with mean EPSC amplitude for three types of connections. Lines indicate linear regression.

Triton X-100 overnight at $4^{\circ} \mathrm{C}$. After washing in TBS, the sections were reacted with $0.02 \%$ diaminobenzidine tetrahydrochloride $(\mathrm{DAB})$ and $0.001 \% \mathrm{H}_{2} \mathrm{O}_{2}$ in TBS. They were postfixed in $1 \% \mathrm{OsO}_{4}$ in $\mathrm{PB}$ containing $7 \%$ glucose, dehydrated, and embedded on glass slides in Epon. Sections used for electron microscopic (EM) observation were postfixed for 60 min in $1 \% \mathrm{OsO}_{4}$ in $\mathrm{PB}$, reacted with $1 \%$ uranyl acetate, then dehydrated and flat-embedded on silicon-coated glass slides in Epon. After reconstruction with Neurolucida, stained cells were serially sectioned at thickness setting $50 \mathrm{~nm}$ using an ultramicrotome (Reichert Ultracut S, Leica Microsystems). The ultrathin sections were mounted on Formvarcoated single-slot grids. EM images of the labeled axon terminals and 


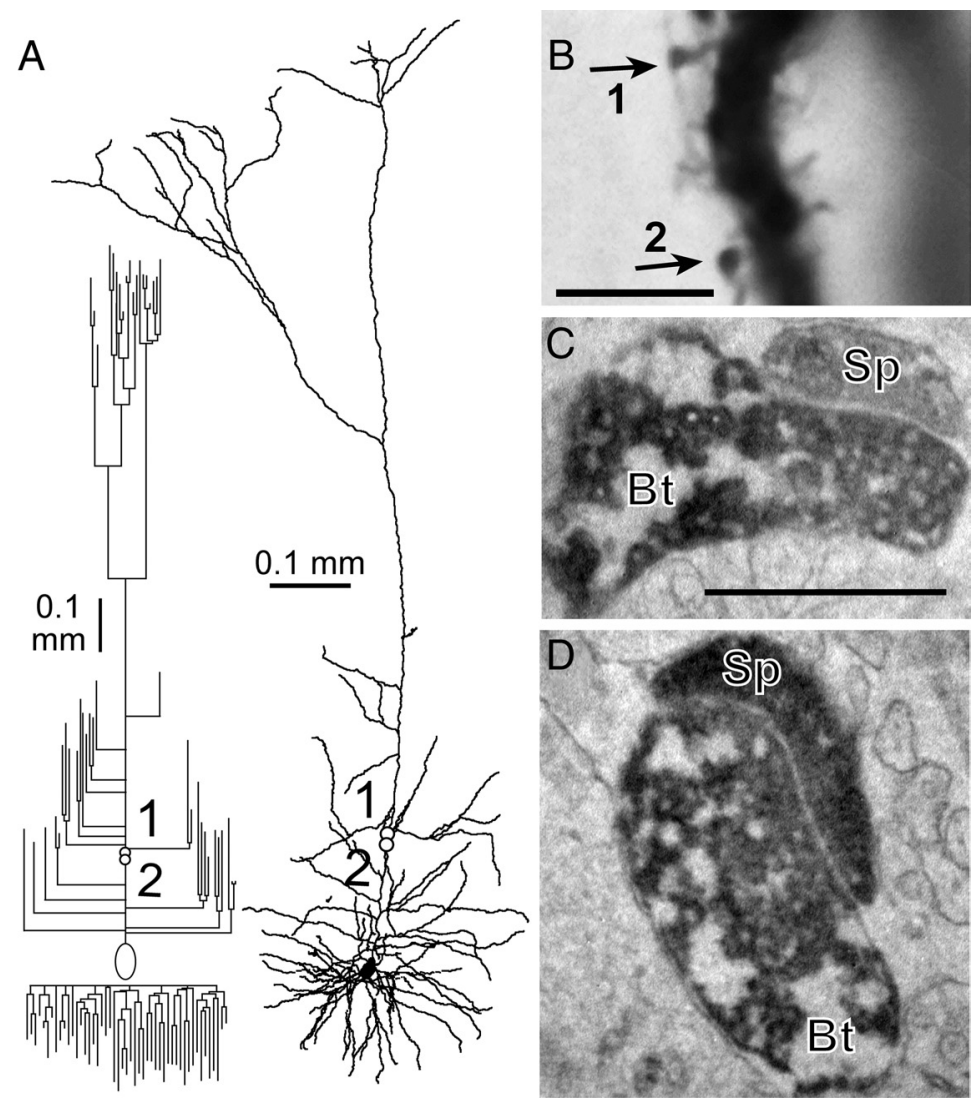

Figure 3. Synapse formation at contact sites in a $\mathrm{CPn} / \mathrm{CPn}$ pair. $A$, Dendritic reconstruction (right) and dendrogram (left) of a postsynaptic $C P n$ cell in a $C P n / C P n$ pair. Open circles numbered 1 and 2, Two contact sites. $B$, Light microscopic photographs of two contact sites, indicated by arrows corresponding to 1 and 2 in $\boldsymbol{A}$. C, D, EM images of the synaptic contact 1 ( $C$ ) and contact 2 (D). Bt, Presynaptic axonal bouton; $S p$, postsynaptic spine. Note that synaptic vesicle accumulation and clear cleft structure are seen at the synaptic junction. Scale bars: $\boldsymbol{B}, 5 \mu \mathrm{m}$; (in $\boldsymbol{C}) \boldsymbol{C}, \boldsymbol{D}, 0.5 \mu \mathrm{m}$.

dendrites were captured using a CCD camera (XR-41, Advanced Microscopy Techniques) equipped to a Hitachi H-7000 electron microscope. The structures were reconstructed using the 3D reconstruction software, Reconstruct (http://synapses.clm.utexas.edu/tools/index.stm).

Immunohistochemistry. Slices were fixed in $4 \%$ paraformaldehyde and $0.2 \%$ picric acid overnight at $4^{\circ} \mathrm{C}$, and resectioned at $50 \mu \mathrm{m}$ thickness. After cryoprotection, the sections were frozen with liquid nitrogen and thawed at room temperature, followed by incubation in TBS containing $1 \% \mathrm{H}_{2} \mathrm{O}_{2}$ for $30 \mathrm{~min}$. Slices were then incubated with a guinea pig antibody against type 2 vesicular glutamate transporter (VGLUT2) (1:2000; AB2251, Millipore Bioscience Research Reagents) in TBS containing $10 \%$ normal goat serum, $2 \%$ bovine serum albumin, and $0.04 \%$ Triton $\mathrm{X}-100$ overnight at $4^{\circ} \mathrm{C}$. Sections were then incubated in biotinconjugated secondary antiserum followed by $\mathrm{ABC}$ complex and staining with $\mathrm{DAB}$, and processed as above.

Layer definition. L5 was divided into an upper VGLUT2-poor sublamina and a lower VGLUT2-rich one (Kubota et al., 2007), reflecting the differences in thalamic input density. We refer to the former as layer $5 \mathrm{a}$ (L5a), and the latter as layer 5b (L5b).

Quantitative morphology. The stained neurons were drawn with a $60 \times$ or $100 \times$ objective combined with an additional $1.25 \times$ magnification and reconstructed three-dimensionally using the Neurolucida system (MBF Bioscience). The reconstructed neurons were quantitatively analyzed with NeuroExplorer (MBF Bioscience). To evaluate the basal dendritic width, we measured the direct distance from the soma centroid to true endings, not endings cut during slice preparation, along horizontal and vertical axes (horizontal and vertical width).

Putative synaptic contacts (contact sites) were identified as a close apposition of an axonal bouton and a postsynaptic dendrite in the same focal plane using a $100 \times$ objective. When the presynaptic axons and postsynaptic dendrites came within $2.5 \mu \mathrm{m}$ of each other (center-to-center), the locations where dendrites were most close were called approach points.

Data are given as mean $\pm \mathrm{SD}$. For statistical comparison of the two groups, we used the nonparametric Mann-Whitney $U$ test, paired $t$ test, Kolmogorov-Smirnov test, and $\chi^{2}$ test. ANOVA was used for confirmation of significant differences among three connections in individual parameters, followed by post hoc Tukey's test. Significance was accepted when $p<0.05$.

\section{Results}

Layer 5 projection subtype- and sublamina-dependent dendritic branching pattern

Previously, we demonstrated that CCS and CPn cells are completely separate neuronal populations in L5 by injecting two different fluorescent retrograde tracers into the pons (labeling CPn cells) and contralateral striatum (labeling CCS cells), and that these two populations can be simultaneously identified in in vitro slice preparations. We found that dendritic morphologies were influenced not only by neuronal subtype (CCS or CPn cells), but also by the cortical depth of a neuron's somata (Morishima and Kawaguchi, 2006). Here we test the hypothesis that depth-dependent neuronal differentiation is related to thalamic input heterogeneity, since L5 of frontal cortex is divided into two sublaminae, L5a and L5b: the latter had denser innervations from the thalamus (Fig. 1A). Therefore, we compared quantitatively the dendritic branching patterns of identified CPn and CCS cells between the different sublaminae (Fig. 1). We analyzed quantitatively the morphology of biocytin-stained CPn ( $n=34$, including 7 cells analyzed previously by Morishima and Kawaguchi, 2006) and CCS cells $(n=27$, including 20 cells used in our previous analysis). We analyzed three dendritic divisions separately: basal dendrites, dendritic tufts in L1, and oblique branches in $\mathrm{L} 2 / 3$. The dendritic parameters of three divisions were compared between L5a and L5b (Fig. $1 B-D$ ).

CPn cells had more elaborate and vertically compact basal dendrites than did CCS cells (Fig. $1 B$ ), and the number of branch points of basal dendrites was smaller in L5a CPn $(32 \pm 5)$ than in L5b CPn cells $(43 \pm 10 ; p<0.01)$, but were larger in L5a CCS $(27 \pm 5)$ than in L5b CCS cells $\left(23 \pm 5\right.$; $p<0.05$; Fig. $\left.1 C_{1}\right)$. CPn neurons in both L5a and L5b CPn had significantly more branch points than did CCS neurons in L5a $(p<0.05)$ and L5b $(p<$ 0.01) (Fig. 1C $_{1}$.

The basal dendrites of L5a CPn cells had a greater horizontal spread than did L5b CPn cells (mean horizontal width: $105 \pm$ $14.3 \mu \mathrm{m}$ in L5a, $94.2 \pm 11.8 \mu \mathrm{m}$ in L5b; $p<0.05$; Fig. $1 C_{2}$ ). The vertical extents of basal dendrites were similar in L5a and L5b CPn cells (mean vertical extent: $63.5 \pm 8.8 \mu \mathrm{m}$ in L5a, $59.3 \pm 8.8$ $\mu \mathrm{m}$ in L5b; Fig. $1 C_{3}$ ). In contrast, L5a CCS cells had greater horizontal and vertical spread than did L5b CCS cells (horizontal: $118 \pm 14 \mu \mathrm{m}$ in L5a and $103 \pm 14 \mu \mathrm{m}$ in L5b, $p<0.05$; vertical: $85.2 \pm 11.5 \mu \mathrm{m}$ in L5a and $73.4 \pm 18 \mu \mathrm{m}$ in L5b, $p<0.05$; Fig. 
$\left.1 C_{2}, C_{3}\right)$. Finally, the basal dendrites of L5a and L5b CPn cells were, overall, horizontally and vertically more compact than were the basal dendrites of L5a CCS ( $p<$ 0.05 ) and L5b CCS cells $(p<0.05)$ (Fig. $\left.1 C_{2}, C_{3}\right)$.

We also observed sublaminar differences in the apical tufts (in L1) and in dendritic branches in L2/3 (Fig. 1D,E). Dendritic length in the tuft was similar between L5a and L5b CPn cells (4533 \pm $1295 \mu \mathrm{m}$ in L5a; $3651 \pm 1218 \mu \mathrm{m}$ in L5b), but was longer in L5a CCS neurons than in L5b CCS cells $(1251 \pm 1289 \mu \mathrm{m}$ in L5a; $648 \pm 345 \mu \mathrm{m}$ in L5b, $p<0.05$; Fig. $1 D)$. L5a and L5b CPn cells had longer tufts than did L5a $(p<0.01)$ and L5b CCS cells $(p<0.05)$, respectively (Fig. $1 D)$.

The length of apical oblique branches in L2/3 was not significantly different between CPn and CCS cells as a whole $(684 \pm 759 \mu \mathrm{m}$ in CPn and $764 \pm 356 \mu \mathrm{m}$ in CCS cells). Oblique branch length in L2/3 was much longer in L5a CPn cells than in L5b CPn cells ( $1346 \pm 639 \mu \mathrm{m}$ in L5a; $125 \pm 158 \mu \mathrm{m}$ in L5b; $p<0.01)$, but was similar between L5a and L5b CCS cells $(877 \pm 397 \mu \mathrm{m}$ in L5a, $689 \pm 322 \mu \mathrm{m}$ in L5b; Fig. $1 E$ ). Finally, L5b CPn cells had shorter L2/3 oblique branches than L5b CCS cells $(p<0.01$; Fig. $1 E)$.

These results demonstrate that the dendritic morphologies of L5 pyramidal neurons depend not only on the projection subtype, but also on the sublaminae in which somata are located. Because thalamic input in L5 is also segregated between sublaminae (Fig. $1 A$ ), these data suggest that differential dendritic morphologies may be specialized in terms of their thalamic input.

\section{EPSC amplitudes and their fluctuations in individual connection types}

Previously, we compared the characteristics of unitary synaptic transmission in pairs of CCS/CCS and CCS/CPn neurons and reported no significant differences (Morishima and Kawaguchi, 2006). We rarely found connections from CPn to CCS cells. Here we compare the unitary EPSC characteristics in $\mathrm{CPn} / \mathrm{CPn}$ cell pairs with those of CCS/CCS and CCS/CPn pairs. We include in this analysis some data from CCS/CCS and CCS/CPn pairs previous analyzed by Morishima and Kawaguchi (2006) (Table 1).

We generated unitary EPSCs by simultaneous recording from CPn or CCS cells identified with retrograde fluorescence labeling. In synaptically connected cases, a presynaptic action potential in a presynaptic neuron elicited a unitary EPSC in the postsynaptic cell (Fig. 2A). The EPSC latency, rise time, and decay time constant were not dependent on the presynaptic or postsynaptic pyramidal cell subtype (Table 1). However, we found differences in the unitary EPSC amplitude that depended upon neuron subtypes ( $p<0.01$, ANOVA). Unitary EPSCs in CPn/CPn pairs $(32.3 \pm 27.4 \mathrm{pA})$ were larger than those of CCS/CCS pairs (17.0 $\pm 13.7 \mathrm{pA} ; p<0.01$, post hoc Tukey's test), and also larger than those of CCS/CPn connections $(14.7 \pm 9.2 \mathrm{pA} ; p<0.05)$ (Fig. 2 B). CCS/CCS and CCS/CPn pairs had similar mean EPSC amplitudes $(p=0.41)$. These data suggest that EPSC amplitude depends on the identity of the presynaptic cell. To determine whether EPSC amplitude also depended upon the sublaminar location, we tested whether the EPSC amplitude was correlated with the sublaminar location of presynaptic or postsynaptic neurons within L5a or L5b (see Materials and Methods; Fig. 1A). However, there were no sublaminar differences in both connections (Table 1).

Previously it was reported that amplitude variation of unitary EPSPs (CV) correlates negatively with EPSP amplitude (Thomson et al., 1993). Although the mean EPSC amplitude of CPn/CPn connections was significantly larger than for CCS/CCS connections, CV was found to be similar between the two groups (Table 1). Therefore, we further tested for correlation of CV with mean EPSC amplitude for each connection type. For both $\mathrm{CPn} / \mathrm{CPn}$ and CCS/CCS connections, CV was negatively correlated with EPSC amplitude [correlation coefficient (c.c.) $=-0.59(p<0.01)$ for $\mathrm{CPn} / \mathrm{CPn}$; c.c. $=-0.66(p<0.01)$ for CCS/CCS; c.c. $=-0.78(p<0.01)$ for $\mathrm{CCS} / \mathrm{CPn}]$; however, the magnitude of the relationship depended upon the type of synaptic connection (Fig. 2C). Compared with $\mathrm{CPn} / \mathrm{CPn}$ connections, CCS/CCS connections showed a steeper relationship between EPSC variability $(\mathrm{CV})$ and amplitude $(p<0.01$, comparison of two regression parameters). This means that synaptic transmission from CPn to CPn cells is more variable for the given EPSC amplitude relative to CCS-to-CCS connections. Assuming a binomial model of synaptic transmission, $\mathrm{CV}$ would be:

$$
\sqrt{[(1-\mathrm{Pr}) /(\mathrm{Nb} \cdot \mathrm{Pr})]},
$$



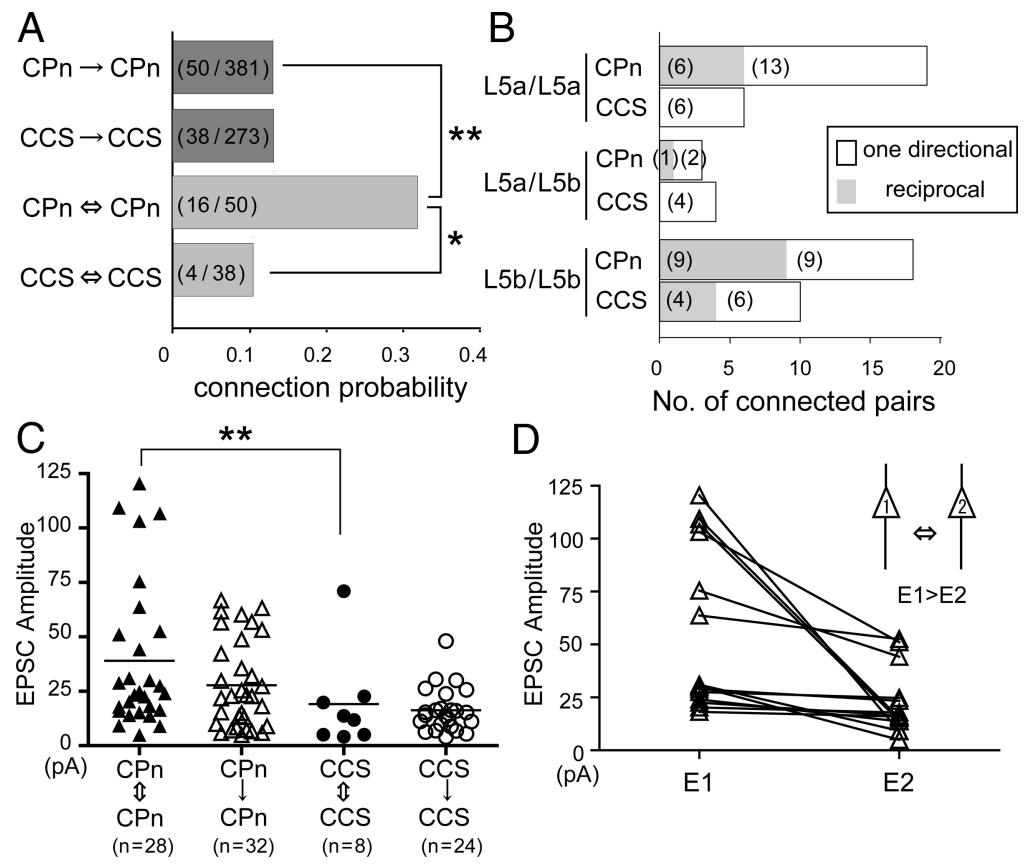

D

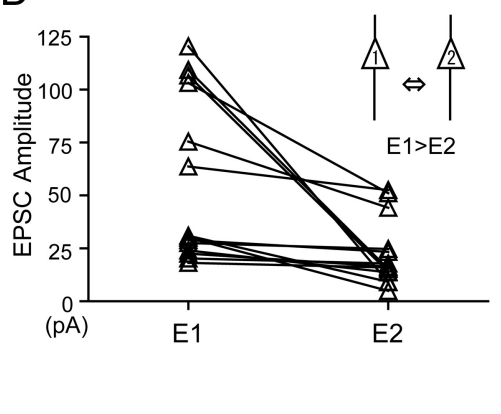

Figure 5. Projection subtype-dependent connection patterns. $\boldsymbol{A}$, Connection probabilities and reciprocity (left). Note higher reciprocal connection probability in CPn pairs than CCS pairs. (n1/n2), [(number of connected pairs)/(number of recorded pairs)]. $\boldsymbol{B}$, Comparison of the reciprocity in L5a and L5b. Gray bars indicate reciprocally connected pair; open bars indicate unidirectional pair. Note that reciprocally connected CCS pairs were found only in L5b. Numbers in parentheses, Pair number. C, Relation of connection patterns and EPSC amplitudes. Note the occurrence of large amplitude of synaptic currents in reciprocally connected CPn pairs. D, Comparison of the larger amplitude (E1) and smaller amplitude (E2) of the reciprocal connections in CPn/CPn pairs. Note that the amplitude $>100 \mathrm{pA}$ was found only in one direction. ${ }^{*} p<0.05 ;{ }^{* *} p<0.01$.

where $\mathrm{Nb}$ is number of release sites; $\mathrm{Pr}$ is release probability (Markram et al., 1997). Therefore, differences in CV for the same EPSC amplitude may be due to fewer release sites and/or lower release probabilities in $\mathrm{CPn} / \mathrm{CPn}$ connections.

\section{Synaptic transmission parameters assuming the binomial model}

The EPSC amplitude and its relation to CV were different among $\mathrm{CPn} / \mathrm{CPn}$ and CCS/CCS connections. To clarify the mechanisms underlying these synaptic differences, we used computer-assisted reconstruction to analyze quantitatively the dendrites and axons of synaptically connected pairs, and identified close appositions made by presynaptic axons and postsynaptic dendrites (putative synaptic contact sites) (Figs. $2 A, 3 A$ ). In a $\mathrm{CPn} / \mathrm{CPn}$ pair we identified two synaptic contacts at the electron microscopic level (Fig. $3 B-D)$. The two axonal boutons made synapses on spines of the CPn cell dendrite. The synaptic junction area of contact 1 was $0.109 \mu \mathrm{m}^{2}$, and that of contact 2 was $0.263 \mu \mathrm{m}^{2}$, based on the $3 \mathrm{D}$ reconstruction. In the following analysis, this connection is distinguished (marked by a gray arrow in Fig. $4 B-F$ ).

The distance of contact sites from soma and their number are expected to influence the amplitude of unitary EPSCs. Contact sites were mapped on the dendrogram of the postsynaptic neurons (Fig. 3A; mean contact number/connection: $3.1 \pm 1.6$ in $17 \mathrm{CPn} / \mathrm{CPn}$ and $2.9 \pm 1.2$ in $13 \mathrm{CCS} / \mathrm{CCS}$ connections). We found contact sites to be distributed mainly on basal dendrites in both CPn/CPn and CCS/CCS connections [basal dendrite contact proportion $=85 \%$ (45 of 53) for CPn/CPn, and 95\% (35 of 37) for CCS/CCS] (Fig. 4A). We further compared the relationship between mean EPSC amplitude and the distance of the putative contact sites (mean distance and the nearest distance from somata) for connections having the same contact site number
$(\mathrm{Nc}=2 ; 10 \mathrm{CPn} / \mathrm{CPn})$. We found no correlation between the contact site distance and mean EPSC amplitude (mean distance: c.c. $=-0.22, p=0.55$; nearest distance: c.c. $=-0.14, p=0.71)$. The distances from soma of contact sites was not significantly different among $\mathrm{CPn} /$ CPn connections $(106 \pm 65 \mu \mathrm{m})$, CCS/ CCS connections $(86 \pm 56 \mu \mathrm{m})$, or CCS/ CPn connections $(97 \pm 60 \mu \mathrm{m} ; p=0.31$, ANOVA) (Fig. 4A). This suggests that contact formation for these three types of connections is similar. Unitary EPSC amplitude was strongly correlated with the number of putative contact sites in both $\mathrm{CPn} / \mathrm{CPn}($ c.c. $=0.92, p<0.01)$ and CCS/ CCS (c.c. $=0.8, p<0.01$ ) connections (Fig. $4 B$ ). However, the dependency of unitary EPSC amplitude on contact site number was dependent upon the type of presynaptic neuron, since the regression slopes were significantly different between the CPn presynaptic and CCS presynaptic connections $(p<0.05)$ (Fig. $4 B)$.

EPSC CV was inversely correlated with the number of contact sites in connections including three types: CPn/CPn, CCS/ CCS, and CCS/CPn connections (c.c. = $-0.4, p<0.05)$. The correlation was significant in CCS/CCS connections (c.c. $=$ $-0.57, p<0.05)$, but not significant in $\mathrm{CPn} / \mathrm{CPn}$ connections (c.c. $=-0.36, p=0.16$; Fig. $4 C$ ). Regression slope was not different between the two types $(p>0.10)$. Combined with the positive correlation found between EPSC amplitude and Nc (Fig. $4 \mathrm{~B}$ ), these correlations suggest that the identified putative contact sites likely reflect functional synaptic release sites.

Assuming both a single release site at individual contacts and the binominal model, $\mathrm{CV}$ would be:

$$
\sqrt{[(1-\mathrm{Pc}) /(\mathrm{Nc} \cdot \mathrm{Pc})]},
$$

where $\mathrm{Nc}$ is contact site number and Pc is release probability at individual contacts. The release probability calculated this way was not significantly different between the connection types $[\mathrm{Pc}=0.64 \pm 0.16$ for $\mathrm{CPn} / \mathrm{CPn}$ connections $(n=17), 0.56 \pm$ 0.18 for CCS/CCS connections $(n=13)$, and $0.53 \pm 0.18$ for CCS/CPn connections $(n=6) ; p=0.26$, ANOVA] (Fig. $4 D$ ). Thus, CV differences at the similar amplitude shown in Figure $2 C$ cannot be explained by lower release probabilities in $\mathrm{CPn} / \mathrm{CPn}$ than in CCS/CCS connections. These findings suggest that the larger relative $\mathrm{CV}$ in $\mathrm{CPn} / \mathrm{CPn}$ connections is better explained by fewer release sites and larger quantal amplitude at individual contacts. Assuming the binominal model (Eq. 3) and mean EPSC amplitude $=\mathrm{Qc} \cdot \mathrm{Nc} \cdot \mathrm{Pc}(\mathrm{Qc}$, quantal current size $)$, Qc would be $18.9 \pm 10.6 \mathrm{pA}$ for $\mathrm{CPn} / \mathrm{CPn}, 9.8 \pm 3.9 \mathrm{pA}$ for $\mathrm{CCS} / \mathrm{CCS}$, and $7.4 \pm 2.1 \mathrm{pA}$ for CCS/CPn $(p<0.01$, ANOVA; Fig. $4 E)$. Consistent with this calculation, the regression slope of the EPSC amplitude against contact number was larger in $\mathrm{CPn} / \mathrm{CPn}$ pairs than in CCS/CCS pairs (Fig. $4 B$ ), while the amplitude per contact in $\mathrm{CPn} / \mathrm{CPn}(11.7 \pm 5.8 \mathrm{pA} /$ contact $)$ was larger than in CCS/CCS $(5.7 \pm 3.1 \mathrm{pA} /$ contact $)$ and CCS/CPn $(3.9 \pm 1.9 \mathrm{pA} /$ contact $)$ pairs $(p<0.01)$ (Fig. $4 F)$. These results suggest that $\mathrm{CPn} / \mathrm{CPn}$ 
connections at individual contact sites are stronger than CCS/CCS connections.

Projection subtype-dependent connectivity Connection probabilities for CCS to CCS cell pairs and CCS to CPn cell pairs are similar, but that for CPn to CCS cells is much lower (Morishima and Kawaguchi, 2006). Here we compared the probabilities of connections in CPn/CPn and CCS/ CCS pairs. The intersoma distance between the connected cells was similar between the pair types $(45.8 \pm 29.4 \mu \mathrm{m}$ in $\mathrm{CPn} / \mathrm{CPn}$ and $57.2 \pm 43.9 \mu \mathrm{m}$ in CCS/ CCS pairs, $p=0.35$ ). Synaptically connected pairs were found with similar probabilities in CPn/CPn (50 of 381 pairs, connection probability $=0.13$ ) and CCS/ CCS pairs ( 38 of 273 pairs, probability $=$ $0.14 ; p=0.86$ ) (Fig. $5 A$ ). However, reciprocal connections were three times more likely to be found in connected CPn pairs ( 16 of 50 connected pairs, reciprocity $=0.32$ ) than in CCS pairs ( 4 of 38 connected pairs, reciprocity $=0.11 ; p<0.05)$. This indicates that the CPn population is composed of subgroups of mutually connected cells in the frontal cortex. We next tested whether reciprocal connectivity in CPn/CPn $(n=40)$ and CCS/CCS $(n=24)$ pairs was influenced by their sublaminar somatic location within L5a and L5b (Fig. 5B). We found reciprocal connections of $\mathrm{CPn} / \mathrm{CPn}$ pairs broadly within L5 [in L5a ( 6 of 19 pairs), L5a/L5b (1 of 3 pairs), and L5b ( 9 of 18 pairs)]. Conversely, CCS/CCS reciprocal connections were found only in L5b/ L5b pairs ( 4 of 10 pairs), with no reciprocal connectivity in pairs spanning L5a and L5b (0 of 6 pairs in L5a and 0 of 4 pairs in L5a/L5b).

Some CPn/CPn connections exhibited larger-amplitude EPSCs than did others (Fig. 2B). To test whether EPSC amplitude is correlated with the direction of connectivity, we compared one way and reciprocally connected CPn/CPn and CCS/CCS pairs (Fig. 5C). EPSC amplitude in reciprocal $\mathrm{CPn} / \mathrm{CPn}$ connections was larger than that found in reciprocal CCS/CCS pairs $(p<$ $0.05)$, but not significantly larger than that found in unidirectional CPn/CPn connections $(p=0.08)$. Very large amplitude EPSCs $(>100 \mathrm{pA}$ ) were found only in one direction of reciprocally connected $\mathrm{CPn} / \mathrm{CPn}$ pairs (Fig. $5 C$ ). In reciprocally connected CPn/CPn pairs, EPSCs of both directions were compared using reciprocal EPSC amplitude ratio (larger/smaller amplitudes). The EPSC amplitude ratio of pairs including currents larger than $100 \mathrm{pA}$ was $7.6 \pm 4.7(n=4)$, but that including only currents smaller than $100 \mathrm{pA}$ was $1.9 \pm 1.5(n=11 ; p<0.05)$ (Fig. 5D). Thus, reciprocally connected $\mathrm{CPn} / \mathrm{CPn}$ pairs included large-amplitude EPSCs, but only in one direction, suggesting their unbalanced synaptic relation.

\section{Relation of reciprocity and axodendritic approaching patterns}

L5 pyramidal cells are mutually connected by their local axon collaterals within L5 (Markram et al., 1997; Morishima and
Kawaguchi, 2006). Reciprocal connections were found more often in CPn/CPn than in CCS/CCS pairs. In CCS/CCS connections, reciprocity was found only in L5b/L5b pairs. These may be related to more local axon branching in CPn cells than in CCS cells, and in L5b than L5a CCS cells. Therefore, we compared the axonal arborization between L5a and L5b (Fig. 6A). In L5a, axon length of L5a CPn cells ( $3493 \pm 2185 \mu \mathrm{m})$ was not different from that of L5b CPn cells $(2151 \pm 1517 \mu \mathrm{m} ; p=0.17$; Fig. $6 B)$. In L5b, axon length of L5a CPn cells $(5602 \pm 1711 \mu \mathrm{m})$ was also not different from that of L5b CPn cells $(7106 \pm 1892 \mu \mathrm{m} ; p=0.07)$. In contrast to CPn cell axonal arborization, L5a CCS cells extended their axons mainly in L5a (7129 $\pm 3945 \mu \mathrm{m}$ in L5a and $2412 \pm 2265 \mu \mathrm{m}$ in L5b; $p<0.01)$, whereas L5b CCS cells did so in L5b $(3745 \pm 2535 \mu \mathrm{m}$ in L5a and 10,422 $\pm 4048 \mu \mathrm{m}$ in L5b; $p<0.01)$. Axonal length of L5a CCS cells within L5a was not different from that of L5a CPn cells ( $p=0.11$; Fig. $6 B)$. L5b CCS cells had longer axons within L5b than did L5b CPn cells $(p<$ $0.05)$. These data indicate that CCS cells have axon collaterals more heavily in the same sublamina as their parent cell body. From these results, axonal morphologies of L5 pyramidal neurons depend not only on the projection subtype, but also on the sublaminae where somata are located.

Since higher reciprocal connectivity may otherwise reflect more frequent overlapping of axon collaterals and dendritic branches between neighboring CPn cells than CCS cells, we examined how morphological differences might influence axodendritic overlap to impact contact formation. We located points in 
A
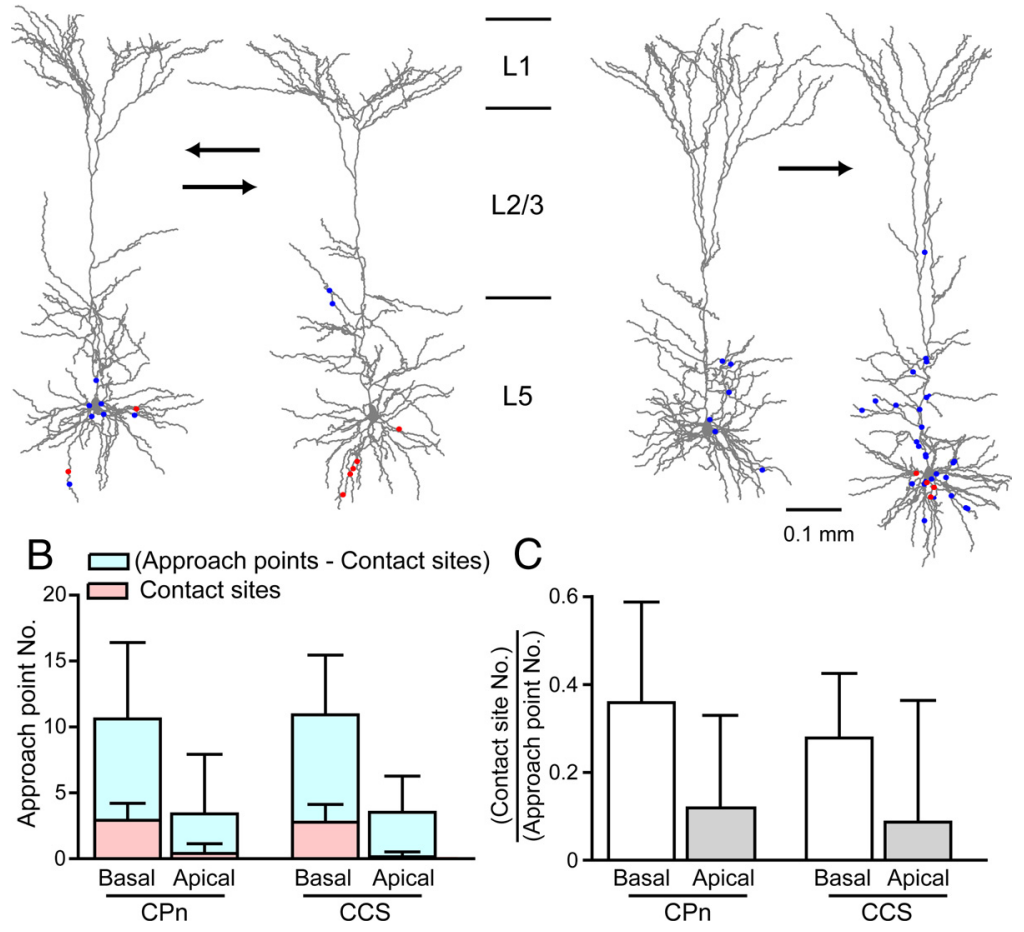

Figure 7. Approach points of potential presynaptic axons and postsynaptic dendrites in CPn/CPn and CCS/CCS pairs. A, Dendritic reconstructions of two pairs of CPn cells. Left, A pair connected reciprocally; right, a pair connected in one way. Red circles indicate contact site; blue circles indicate approach point without contact. $\boldsymbol{B}$, Comparison of approach point and contact site number in basal and apical dendrites of CPn/CPn $(n=15)$ and CCS/CCS pairs $(n=13)$, respectively. C, Ratio of contact sites to total approach points in basal and apical dendrites of $\mathrm{CPn} / \mathrm{CPn}$ and CCS/CCS pairs, respectively. Error bar, SD.

the dendritic trees of connected pairs, where axon collaterals of the other cell come within $2.5 \mu \mathrm{m}$ of the dendritic shaft [approach points; potential contact sites by spine elongation (Morishima and Kawaguchi, 2006)]. Approach points included contact sites (close appositions of an axonal bouton and a postsynaptic dendrite). We then mapped contact sites and approach points on dendritic reconstructions of pairs of cells (Fig. 7A; left, reciprocally connected pair; right, one-directional pair). The number of approach points in CPn pairs (10.6 \pm 6.4 on basal and $3.4 \pm 4.6$ on apical dendrites, $n=15$ ) was similar to that found in CCS pairs $(10.9 \pm 5$ on basal and $3.5 \pm 2.8$ on apical dendrites, $n=13$ ) (Fig. $7 B$ ).

To relate contact formation with axodendritic approaching, we compared the ratio of the number of putative contact sites to the total approach points (putative contact sites/total approach points) between CPn/CPn and CCS/CCS connections. The contact site proportions were similar between $\mathrm{CPn} / \mathrm{CPn}(0.36 \pm 0.23$ on basal and $0.12 \pm 0.21$ on apical dendrites) and CCS/CCS connections $(0.28 \pm 0.15$ on basal and $0.09 \pm 0.28$ on apical dendrites) (Fig. 7C). We also compared the number of approach points in L5a and L5b, but found no significant differences. These results suggest that spatial configurations of presynaptic axons and postsynaptic dendrites are similar between $\mathrm{CPn} / \mathrm{CPn}$ and CCS/CCS cells, and that the higher reciprocity found in $\mathrm{CPn} / \mathrm{CPn}$ pairs reflects synaptic selectivity rather than morphological differences in dendritic and axonal extents.

\section{Connection type-specific short-term plasticity}

Finally, we investigated the short-term plasticity of CPn/CPn, CCS/CCS, and CCS/CPn connections, by comparing amplitudes of successive EPSCs (EPSC1 and EPSC2) induced by two presyn- aptic action potentials elicited at $100 \mathrm{~ms}$ intervals (Fig. 8A). To compare relation of EPSC1 amplitudes and paired-pulse ratios, we plotted means of EPSC2 against those of EPSC1 (CPn/CPn, $n=60$; CCS/ CCS, $n=22$; CCS/CPn, $n=7$; Fig. $8 B$ ). The regression line was significantly different between the CPn/CPn and CCS/ CCS connections $(p<0.01$, comparison of two regression parameters). Even CPn/ CPn connections with larger EPSC1 showed paired-pulse facilitation (pairedpulse ratios $>1$ ). The paired-pulse ratio (EPSC2/EPSC1; average of $>20$ responses) was larger in CPn/CPn (1.27 \pm $0.34)$ than in CCS/CCS $(0.87 \pm 0.34 ; p<$ 0.01 , post hoc Tukey's test) or CCS/CPn $(0.85 \pm 0.05 ; p<0.01)$ connections. Facilitating responses were more often found in $\mathrm{CPn} / \mathrm{CPn}$ (46 of 60 pairs) than in CCS/CCS ( 4 of $22 ; p<0.01$ ) or CCS/CPn (0 of 7 ) connections (Fig. $8 C$ ). CPn/CPn connections showed similar facilitation in both sublaminae [L5a: $1.24 \pm 0.36(n=$ 25); L5b: $1.28 \pm 0.31(n=25) ; p=0.43$ ]. Paired-pulse ratios were correlated with EPSC1 amplitude variability (CV) [c.c. $=$ $0.64(p<0.01), n=60$ in CPn/CPn; c.c. $=0.78(p<0.01) n=22$ in CCS/CCS $]$. Interestingly, initial EPSCs (EPSC1s) with facilitating responses had larger CVs than did paired EPSCs (EPSC2s). In contrast, EPSC1s with depressing responses had smaller CVs than did EPSC2s. Indeed, CV increased from EPSC1 to EPSC2 in CCS/ CCS (ratio, $1.24 \pm 0.40, n=22)$ and CCS/CPn $(1.38 \pm 0.47, n=$ 7) pairs, but decreased in CPn/CPn pairs $(0.79 \pm 0.26, n=60$; $p<0.01$ ). The amplitude ratio of EPSC2 to EPSC1 (paired-pulse ratio) was negatively correlated with the $\mathrm{CV}$ ratio (Fig. $8 \mathrm{C}$ ). The $\mathrm{CV}$ ratio of facilitating synapses was $<1$ (Fig. $8 C$ ). In addition, when the ratio of $1 / \mathrm{CV}^{2}$ was plotted against the paired-pulse ratio (Malinow and Tsien, 1990; Fig. 8D), the ratio of $1 / \mathrm{CV}^{2}$ for facilitating responses was generally larger than the identity line (having a slope of 1), whereas depressing responses had the ratio of $1 / \mathrm{CV}^{2}$ values smaller than the identity line. These observations indicate that paired-pulse ratio depends mostly upon the subtype of presynaptic pyramidal neuron.

\section{Relation of short-term plasticity and release probability}

Using a binomial model of synaptic transmission and the $\mathrm{CV}$ equation (Eq. 3), we calculated the release probabilities for EPSC1 (Pc1) and EPSC2 (Pc2) for both facilitating and depressing responses in morphologically reconstructed connections (Fig. 9A). Pc2 of facilitating response was found to be significantly larger than Pc1 ( $p<0.01, n=15$ in CPn/CPn and $n=2$ in CCS/CCS), while Pc2 of depressing response was significantly smaller than Pc1 ( $p<0.05, n=2$ in CPn/CPn, $n=6$ in CCS/ CCS, and $n=4$ in CCS/CPn) (Fig. 9B), further suggesting that paired-pulse facilitation and depression may be caused by increased or decreased release probability, respectively. We next compared Qc in facilitating and depressing responses. Qc2 and Qc1 of facilitating responses were not significantly different $(p=$ 0.27, $n=15$ in CPn/CPn, $n=2$ in CCS/CCS), but Qc2 of depressing responses was smaller than that of Qc1 $(p<0.01, n=2$ 
in CPn/CPn, $n=6$ in CCS/CCS, $n=4$ in CCS/CPn) (Fig. 9C). Release probability ratio was well correlated with the pairedpulse ratio [c.c. $=0.76(p<0.01)$ in $n=$ 17 in CPn/CPn, $n=8$ in CCS/CCS, $n=4$ in CCS/CPn] (Fig. 9D). This correlation was found also within individual subtypes [c.c. $=0.75(p<0.01)$ in $\mathrm{CPn} / \mathrm{CPn}(n=$ $17)$; c.c. $=0.94(p<0.01)$ in $\mathrm{CCS} / \mathrm{CCS}$ $(n=8)]$. These results suggest that changes of the release probability could mostly explain those of the paired-pulse ratio, especially in facilitating responses.

Paired-pulse ratio was correlated with release probability in individual connections: lower paired-pulse ratios coincided with higher release probabilities (Fig. 9E; c.c. $=-0.67$ and $p<0.01$ for $\mathrm{CPn} / \mathrm{CPn}$; c.c. $=-0.87$ and $p<0.05$ for CCS/CCS; c.c. $=-0.02$ and $p=0.99$ for CCS/CPn). The paired-pulse ratios for $\mathrm{CPn} / \mathrm{CPn}$ pairs were larger than those for CCS/CCS and CCS/CPn pairs $(p<0.01)$. At the similar release probability, however, those of $\mathrm{CPn} / \mathrm{CPn}$ connections were higher than CCC/CCS and CCS/CPn connections (Fig. 9E). These suggest that temporal characteristics of successive presynaptic spikes among CPn and CCS cell connections are determined by both the presynaptic pyramidal subtype and release probability at individual connections.

To further confirm that the temporal characteristics of successive synaptic inputs are strongly influenced by the presynaptic pyramidal cell subtype, we applied the phenomenological model to successively induce EPSCs from CPn or CCS cells (Markram et al., 1998; see Material and Methods; Fig. 10A). From this model, we obtained $U$ (baseline level of fraction of resources $u$, analogous to the release probability), $\tau_{\mathrm{D}}$ (recovery time constant from depression), and $\tau_{\mathrm{F}}$ (recovery time constant from facilitation). $U$ was not significantly different between CPn/CPn $(0.37 \pm 0.14, n=$ $10)$ and CCS/CCS pairs $(0.41 \pm 0.19, n=7, p=0.76)$. However, $\tau_{\mathrm{D}}$ was $317 \pm 146 \mathrm{~ms}$ in CPn/CPn pairs $(n=10)$ and $532 \pm 244$ $\mathrm{ms}$ in CCS/CCS pairs $(n=7 ; p=0.05)$, while $\tau_{\mathrm{F}}$ was $519 \pm 981$ $\mathrm{ms}$ in $\mathrm{CPn} / \mathrm{CPn}(n=10)$ and $65 \pm 110 \mathrm{~ms}$ in CCS/CCS pairs $(n=$ $7 ; p<0.05)$. These corresponded to more facilitation in $\mathrm{CPn} /$ CPn pairs and depression in CCS/CCS pairs. The paired-pulse ratio (EPSC2/EPSC1) was larger in CPn/CPn $(1.04 \pm 0.34, n=$ $10)$ than in CCS/CCS pairs $(0.65 \pm 0.18, n=7, p<0.01)$, and was not significantly correlated with $U$, a parameter closely related to the release probability of initial EPSCs (Fig. $10 B$; c.c. $=-0.50, p=$ 0.15 for CPn/CPn pairs; c.c. $=-0.156, p=0.75$ for CCS/CCS pairs). For a similar $U, \mathrm{CPn} / \mathrm{CPn}$ connections exhibited higher paired-pulse ratios than CCC/CCS connections. These results support the idea that the synaptic temporal characteristics are strongly influenced by the presynaptic pyramidal cell subtype, even among connections with similar release probabilities. paired-pulse amplitude ratio.
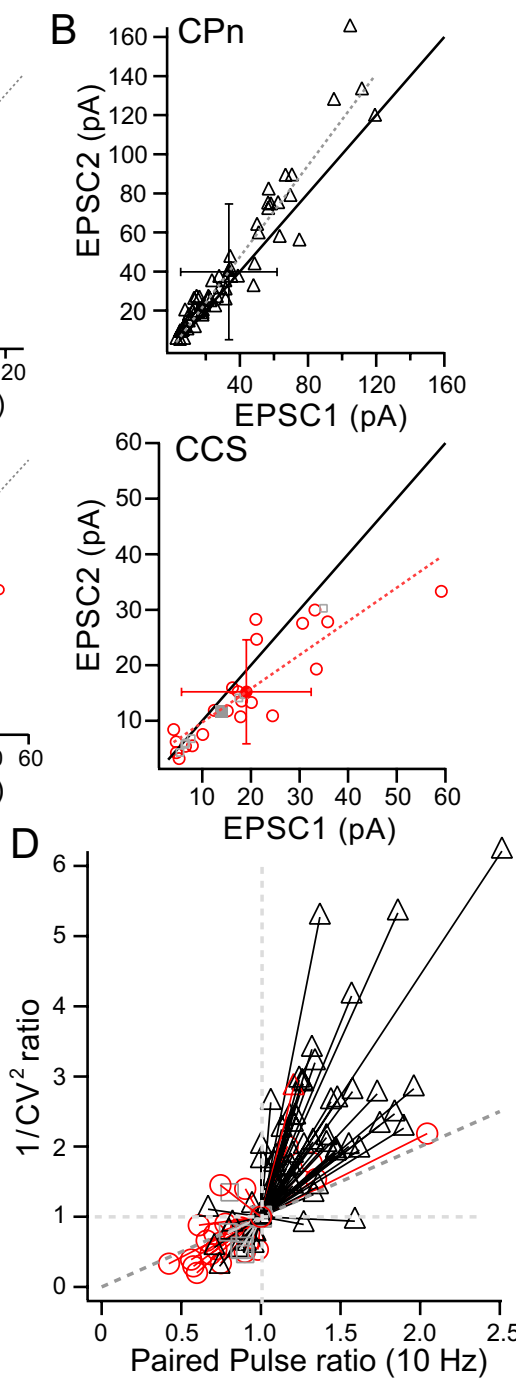

Paired Pulse ratio $(10 \mathrm{~Hz})$
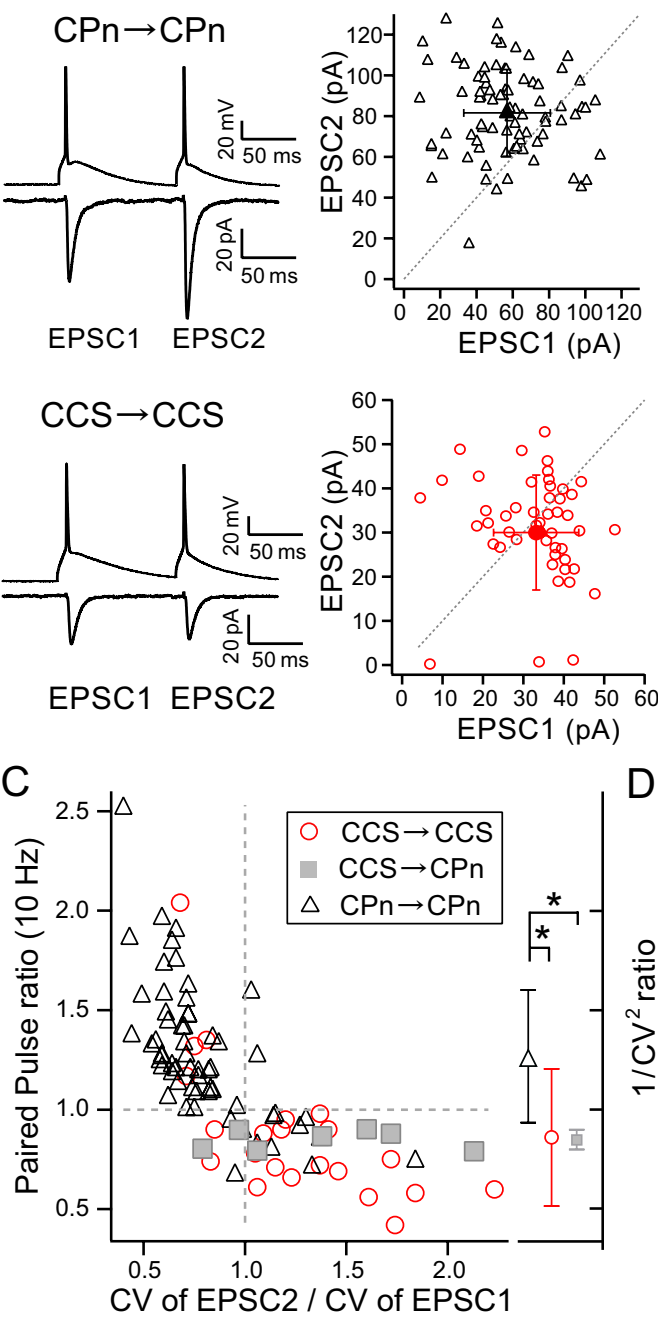

Figure 8. Connection type-specific short-term plasticity. $A$, Averaged paired-pulse responses (EPSC2/EPSC1) induced by two successive presynaptic firings (100 ms interval) in a $\mathrm{CPn} / \mathrm{CPn}$ connection (paired-pulse ratio $=1.44$ ) and a $\mathrm{CCS} / \mathrm{CCS}$ connection Filled symbol, mean \pm SD; dotted line, line with unit slope. $B$, Mean of EPSC2 amplitudes against that of EPSC1 amplitudes in each (ion. Filled symbol, mean \pm SD of all connections. The slope of a fitted line (solid line) was 1.17 for CPn cells and 0.61 for CCS was primarily $<1$, whereas that of depression responses was $>1$. Dotted line, Line with unit slope. $\boldsymbol{D}$, Relation of $1 / \mathrm{CV}^{2}$ ratio with

\section{Discussion}

We have been characterizing the diversity and connection specificity of L5 pyramidal neurons in the rat frontal cortex (Morishima and Kawaguchi, 2006; Otsuka and Kawaguchi, 2008). Here, we report that two pyramidal neuron projection subtypes, CPn and CCS, are highly differentiated in their morphologies and synaptic connectivity and transmission based on their sublaminar location within L5 (Fig. 11). Dendritic and axonal branching patterns were dependent on cell subtype as well as on sublaminar position of the cell body. CPn and CCS pairs had similar connection probabilities, but had subtype-specific probabilities for synaptic reciprocity, synaptic strength, and short-term plasticity.

Dendritic branching patterns were different between CPn and CCS cells, but also varied within the same subtype according to the sublamina (L5a or L5b) in which the soma was located. Because thalamic relay nuclei participating in the basal ganglia and cerebellum pathways innervate different sublaminae of L5 in the 


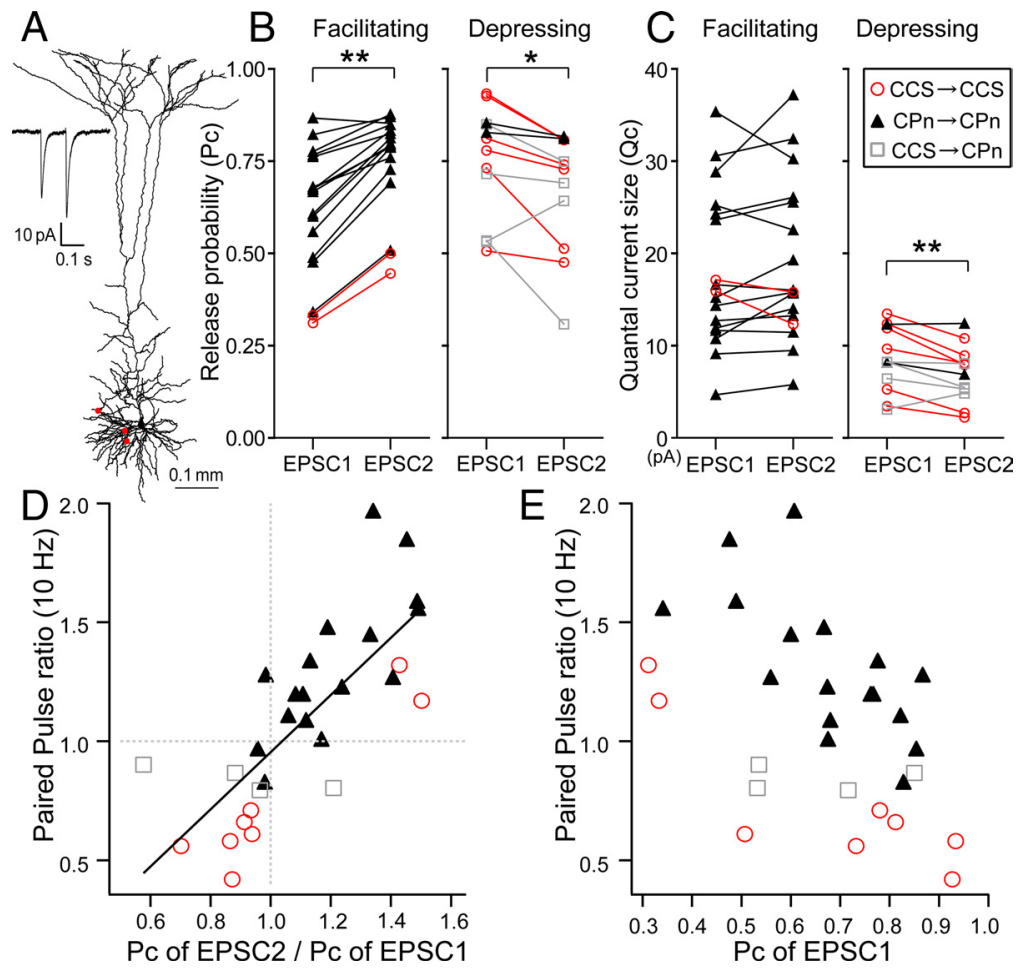

Figure 9. Relation of paired-pulse ratio to release probabilities assuming the binomial model. $\boldsymbol{A}$, Reconstruction of postsynaptic dendrites and contact sites (red circles) of a CPn cell innervated by another (Pn cell, with facilitating type of transmission shown in inset. $\boldsymbol{B}$, Comparison of release probability (PC) of EPSC1 and EPSC2 obtained by assuming a binominal model for facilitating and depression synapses. Note release probability increase in facilitating responses and decrease in depressing responses. $\boldsymbol{C}$, Comparison of quantal current size (QC) of EPSC1 and EPSC2 obtained by assuming a binominal model in facilitating and depression synapses. $D$, Relation of paired-pulse responses with release probability ratio (PC of EPSC2/PC of EPSC1). Note their strong correlation. $\boldsymbol{E}$, Relation of paired-pulse ratio to release probability of initial EPSCs. Note that $C \mathrm{Pn} / \mathrm{CPn}$ pairs had larger paired-pulse ratio at the same release probability. In $\boldsymbol{B}$ and $\boldsymbol{C},{ }^{*} p<0.05 ;{ }^{* *} p<0.01$ (paired $t$ test).

frontal cortex (Jones, 2001, 2007; Kuramoto et al., 2009; RubioGarrido et al., 2009), L5 pyramidal neurons in different sublaminae likely receive differential thalamic drive. The basal ganglia and cerebellar pathways go to the rostromedial and caudolateral parts of the ventral anterior (VA) and ventral lateral (VL) thalamic nuclei (VA-VL complex), respectively, from which the former send axons to L1, while the latter project to middle cortical layers (Jones, 2001, 2007; Kuramoto et al., 2009; Rubio-Garrido et al., 2009). We found dendritic branching differences of CPn and CCS cells to be correlated with the sublaminar distributions of the thalamic inputs, suggesting that L5 pyramidal projection neurons are specialized according to their participation in corticobasal ganglia and corticocerebellar loops.

Selective excitatory synapse formation on specific targets has been reported in several cortical areas (Kozloski et al., 2001; Mercer et al., 2005; Song et al., 2005; Yoshimura et al., 2005; Kampa et al., 2006; Le Bé et al., 2007; Thomson and Lamy, 2007; Brown and Hestrin, 2009). However, selective reciprocal connections between pyramidal cells have been described only in the ferret medial prefrontal cortex (Wang et al., 2006). For the first time, we report higher than expected reciprocity in an identified projection cell subtype (CPn cells). We found this cell type-specific increase in reciprocity even though axodendritic overlap was similar among the projection subtypes. Thus, selective intralaminar connectivity may generate vertically oriented projection-specific subnetworks in the frontal cortex similar to input-specific functional columns in sensory cortex.

In addition to projection-specific connectivity, we found that synaptic transmission from presynaptic CPn cells was stronger than that from presynaptic CCS cells. EPSC amplitudes per individual contact were larger in $\mathrm{CPn} / \mathrm{CPn}$ connections than in CCS/CCS and CCS/CPn connections. Consistent with the relationship between unitary current and contact site number, quantal current size was larger in $\mathrm{CPn} /$ CPn connections than in CCS/CCS and CCS/CPn connections, assuming a binomial model. Thus, the synaptic strength between L5 pyramidal cells is dependent on the presynaptic projection subtype. Differences in quantal current may reflect presynaptic terminal differentiation. Previous reports have demonstrated that $\mathrm{CPn}$ terminals in the striatum have larger synaptic boutons than do the terminals of CCS neurons (Reiner et al., 2003; Lei et al., 2004). This suggests that CPn neurons may act as "driver cells" whose axons are specialized to deliver stronger excitatory input to both intra- and extracortical targets (Sherman and Guillery, 1998).

Larger EPSCs $(>100 \mathrm{pA})$ were found in a few reciprocally connected pairs. Interestingly, one direction amplitude was much larger than the other. In the somatosensory cortex, large amplitudes of unitary EPSPs were rarely found, but such large EPSPs with sparse connections could greatly contribute to overall network activity (Lefort et al., 2009). When a given CPn cell capable of generating large amplitude was excited, that cell subsequently would drive other CPn cells within the mutually connected module. This suggests a further hierarchical structure within the CPn module.

The temporal characteristics of EPSCs depend on their amplitude (Thomson et al., 1993). We revealed that, in addition to EPSC amplitudes, synaptic temporal characteristics depend on the extracortical projection pattern of presynaptic pyramidal cells. CPn/CPn connections were facilitatory even at larger EPSC amplitudes, whereas CCS/CCS and CCS/CPn connections showed depression. This new finding indicates that the frequency characteristics of information coding are differentiated according to the output type. These observations are consistent with temporal facilitation observed in some connections between thick tufted pyramidal cells (Wang et al., 2006). Facilitating excitatory synaptic transmission between reciprocally connected CPn neurons may selectively increase the gain of output to the brainstem.

Our data reveal that changes in release probability are strongly correlated with the paired-pulse response. Paired-pulse facilitation in $\mathrm{CPn} / \mathrm{CPn}$ connections is enhanced by increased release probability, while depression at CCS/CCS connections is correlated with decreased release probability. Even when connections have similar initial release probabilities, the paired-pulse response of CPn/CPn pairs is more facilitatory than those of CCS/ CCS and CCS/CPn pairs. These findings were further supported by analysis based on the phenomenological approach (Markram et al., 1998). The paired-pulse ratio was not significantly dependent on the utilization parameter $U$ (fractions of synaptic transmission resources used by a spike; closely related to the release probability). 
A

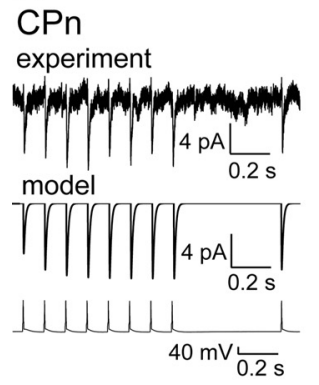

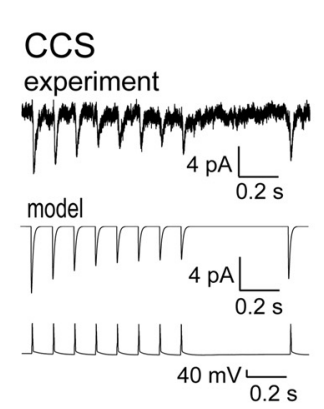

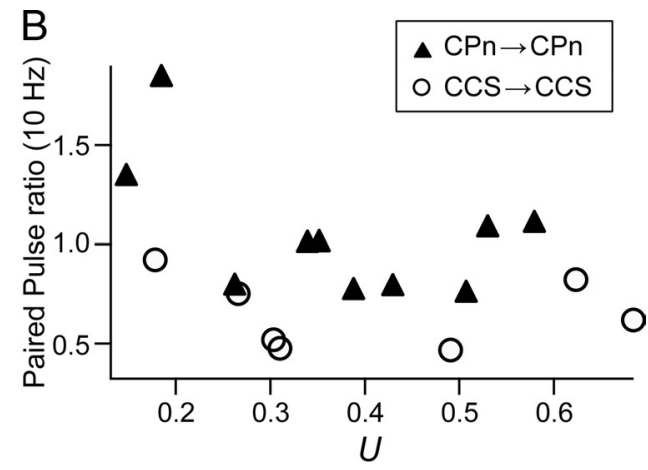

Figure 10. Relation of paired-pulse ratio to the fraction of resources used by a spike based on the phenomenological approach. $\boldsymbol{A}$, Left, EPSCs induced in a (Pn cell (top trace) by a train of presynaptic CPn cell spikes (bottom trace; 8 spikes at $10 \mathrm{~Hz}$, followed by a spike at $500 \mathrm{~ms}$ later) and the simulated currents based on the phenomenological model (middle). Model parameter: $U=$ $0.58 ; \tau_{\mathrm{D}}=34.8 \mathrm{~ms} ; \tau_{\mathrm{F}}=702 \mathrm{~ms}$. Paired-pulse ratio at $10 \mathrm{~Hz}=1$.1. Right, EPSCs induced in a CCS cell by a train of presynaptic CCS cell spikes and the simulated currents. Model parameter: $U=$ $0.27 ; \tau_{\mathrm{D}}=480 \mathrm{~ms} ; \tau_{\mathrm{F}}=2.8 \mathrm{~ms}$. Paired-pulse ratio at $10 \mathrm{~Hz}=0.75$. B, Relation of paired-pulse ratio to parameter $U$ corresponding to the release probability at the baseline level.

Thus, the synaptic temporal characteristic is strongly influenced by the L5 presynaptic projection subtype.

Finally, CPn cells preferentially fire doublets of action potentials and exhibit less spike-frequency adaptation during periods of sustained depolarization (Wang and McCormick, 1993; Kasper et al., 1994; Molnár and Cheung, 2006; Morishima and Kawaguchi, 2006; Hattox and Nelson, 2007; Otsuka and Kawaguchi, 2008). CPn subgroups could amplify their spiking activities using the temporal properties of facilitation, higher reciprocal connectivity, and their intrinsic firing properties. Persistent firing during working memory in the frontal cortex is thought to underlie working memory (Wang, 1999). The synaptic properties and interconnectedness of CPn pairs described here suggest these neurons may provide a suitable substrate for generating persistent depolarization by recurrent excitation. Involvement of frontal cortical CPn cells relaying cortical outputs to the cerebellum in the persistent activity of working memory may be related to cerebellar internal models reproducing the essential properties of movement and mental representations (Ito, 2008).

\section{References}

Alexander GE, DeLong MR, Strick PL (1986) Parallel organization of functionally segregated circuits linking basal ganglia and cortex. Annu Rev Neurosci 9:357-381.

Brown SP, Hestrin S (2009) Intracortical circuits of pyramidal neurons reflect their longrange axonal targets. Nature 457:1133-1136.

Feldmeyer D, Egger V, Lübke J, Sakmann B (1999) Reliable synaptic connections between pairs of excitatory layer 4 neurones within a single 'barrel' of developing rat somatosensory cortex. J Physiol 521:169-190.

Gabbott PL, Warner TA, Jays PR, Salway P, Busby SJ (2005) Prefrontal cortex in the rat: projections to subcortical autonomic, motor, and limbic centers. J Comp Neurol 492:145-177.

Hattox AM, Nelson SB (2007) Layer V neurons in mouse cortex projecting to different targets have distinct physiological properties. J Neurophysiol 98:3330-3340.
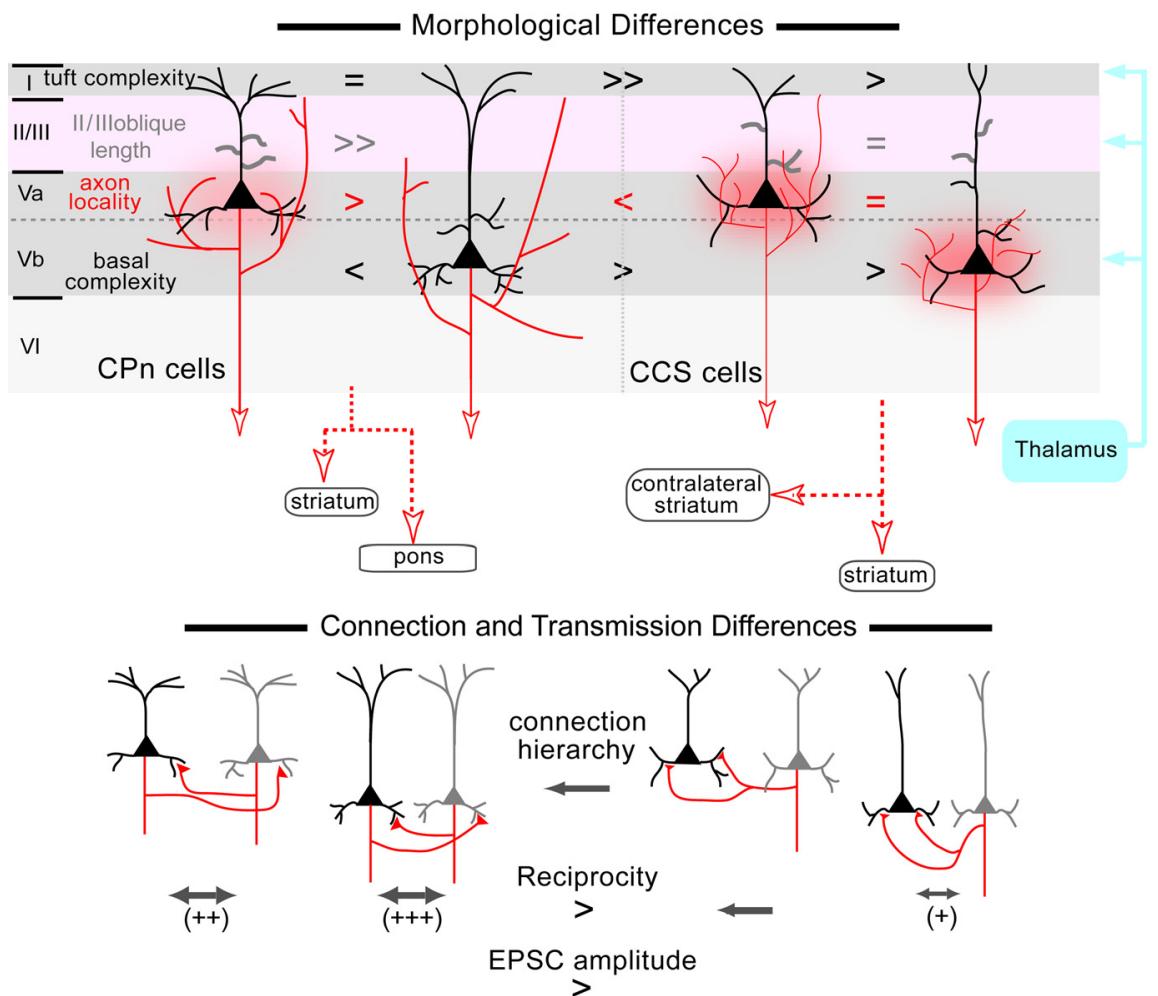

facilitation Short term plasticity depression

Figure 11. Schematicsummary of subtype-dependent morphologies and connectivity. Compared with CCS cells, the apical tufts of CPn neurons are more elaborate within the superficial thalamic inputzone of layer 1 . The tuft dendritic length was similar between $L 5 \mathrm{a}$ and $L \mathrm{~L} b$ CPn cells, but was longer in L5a than in L5b CCS cells. L2/3 oblique branches in the middle thalamic input zone were much fewer in $L 5 b C P n$ cells than $L 5 a$ CPn cells and L5b CCS cells. The basal dendrites of $L 5 b$ CPn cells in the deep thalamic zone were most elaborate. Finally, CCS cells extended axon collaterals more locally, whereas $L 5 b C P n$ cells have the widest distribution of axon collaterals. CPn pairs were connected more reciprocally than CCS Cells. Reciprocal connections were only found in L5b among CCS pairs. The EPSC amplitude was larger in CPn than in CCS connections. Especially, one direction of reciprocally connected pairs sometimes showed large EPSCs (>100 pA). CPn pairs showed facilitation to successive presynaptic spikes, whereas CCS pairs showed depression.

Ito M (1984) The cerebellum and neural control. New York: Raven.

Ito M (2008) Control of mental activities by internal models in the cerebellum. Nat Rev Neurosci 9:304-313.

Jones EG (1984) Laminar Distribution of output cells. In: Cerebral cortex (Peters A, Jones E, eds), pp 521-553. New York: Plenum.

Jones EG (2001) The thalamic matrix and thalamocortical synchrony. Trends Neurosci 24:595-601.

Jones EG (2007) The thalamus, Ed 2. Cambridge, UK: Cambridge UP. Kampa BM, Letzkus JJ, Stuart GJ (2006) Cortical feed-forward networks for 
binding different streams of sensory information. Nat Neurosci 9:1472-1473.

Kasper EM, Larkman AU, Lübke J, Blakemore C (1994) Pyramidal neurons in layer 5 of the rat visual cortex. I. Correlation among cell morphology, intrinsic electrophysiological properties, and axon targets. J Comp Neurol 339:459-474.

Kawaguchi Y (1997) Neostriatal cell subtypes and their functional roles. Neurosci Res 27:1-8.

Kawaguchi Y (2003) Local circuit neurons in the frontal cortico-striatal system. In: Excitatory-inhibitory balance: synapses, circuits, and systems (Hensch TK, Figiolini M, eds), pp 125-148. New York: Kluwer Academic/Plenum.

Kozloski J, Hamzei-Sichani F, Yuste R (2001) Stereotyped position of local synaptic targets in neocortex. Science 293:868-872.

Kubota Y, Hatada S, Kondo S, Karube F, Kawaguchi Y (2007) Neocortical inhibitory terminals innervate dendritic spines targeted by thalamocortical afferents. J Neurosci 27:1139-1150.

Kuramoto E, Furuta T, Nakamura KC, Unzai T, Hioki H, Kaneko T (2009) Two types of thalamocortical projections from the motor thalamic nuclei of the rat: a single neuron-tracing study using viral vectors. Cereb Cortex 19:2065-2077.

Le Bé JV, Silberberg G, Wang Y, Markram H (2007) Morphological, electrophysiological, and synaptic properties of corticocallosal pyramidal cells in the neonatal rat neocortex. Cereb Cortex 17:2204-2213.

Lefort S, Tomm C, Floyd Sarria JC, Petersen CC (2009) The excitatory neuronal network of the $\mathrm{C} 2$ barrel column in mouse primary somatosensory cortex. Neuron 61:301-316.

Lei W, Jiao Y, Del Mar N, Reiner A (2004) Evidence for differential cortical input to direct pathway versus indirect pathway striatal projection neurons in rats. J Neurosci 24:8289-8299.

Malinow R, Tsien RW (1990) Presynaptic enhancement shown by whole-cell recordings of long-term potentiation in hippocampal slices. Nature 346: $177-180$.

Markram H, Lübke J, Frotscher M, Roth A, Sakmann B (1997) Physiology and anatomy of synaptic connections between thick tufted pyramidal neurones in the developing rat neocortex. J Physiol 500:409-440.

Markram H, Wang Y, Tsodyks M (1998) Differential signaling via the same axon of neocortical pyramidal neurons. Proc Natl Acad Sci U S A 95:5323-5328.

Mercer A, West DC, Morris OT, Kirchhecker S, Kerkhoff JE, Thomson AM (2005) Excitatory connections made by presynaptic cortico-cortical pyramidal cells in layer 6 of the neocortex. Cereb Cortex 15:1485-1496.

Middleton FA, Strick PL (2000) Basal ganglia and cerebellar loops: motor and cognitive circuits. Brain Res Brain Res Rev 31:236-250.

Molnár Z, Cheung AF (2006) Towards the classification of subpopulations of layer V pyramidal projection neurons. Neurosci Res 55:105-115.

Mongillo G, Barak O, Tsodyks M (2008) Synaptic theory of working memory. Science 319:1543-1546.
Morishima M, Kawaguchi Y (2006) Recurrent connection patterns of corticostriatal pyramidal cells in frontal cortex. J Neurosci 26:4394-4405.

Otsuka T, Kawaguchi Y (2008) Firing-pattern-dependent specificity of cortical excitatory feed-forward subnetworks. J Neurosci 28:11186-11195.

Reiner A, Jiao Y, Del Mar N, Laverghetta AV, Lei WL (2003) Differential morphology of pyramidal tract-type and intratelencephalically projecting-type corticostriatal neurons and their intrastriatal terminals in rats. J Comp Neurol 457:420-440.

Rubio-Garrido P, Pérez-de-Manzo F, Porrero C, Galazo MJ, Clascá F (2009) Thalamic input to distal apical dendrites in neocortical layer 1 is massive and highly convergent. Cereb Cortex 19:2380-2395.

Sato TR, Svoboda K (2010) The functional properties of barrel cortex neurons projecting to the primary motor cortex. J Neurosci 30:4256-4260.

Sherman SM, Guillery RW (1998) On the actions that one nerve cell can have on another: distinguishing "drivers" from "modulators." Proc Natl Acad Sci U S A 95:7121-7126.

Shipp S (2007) Structure and function of the cerebral cortex. Curr Biol 17:R443-R449.

Song S, Sjöström PJ, Reigl M, Nelson S, Chklovskii DB (2005) Highly nonrandom features of synaptic connectivity in local cortical circuits. PLoS Biol 3:e68.

Strick PL, Dum RP, Fiez JA (2009) Cerebellum and nonmotor function. Annu Rev Neurosci 32:413-434.

Thomson AM, Lamy C (2007) Functional maps of neocortical local circuitry. Front Neurosci 1:19-42.

Thomson AM, Deuchars J, West DC (1993) Large, deep layer pyramidpyramid single axon EPSPs in slices of rat motor cortex display paired-pulse and frequency-dependent depression, mediated presynaptically and selffacilitation, mediated postsynaptically. J Neurophysiol 70:2354-2369.

Tsodyks MV, Markram H (1997) The neural code between neocortical pyramidal neurons depends on neurotransmitter release probability. Proc Natl Acad Sci U S A 94:719-723.

Wang XJ (1999) Synaptic basis of cortical persistent activity: the importance of NMDA receptors to working memory. J Neurosci 19:9587-9603.

Wang Y, Markram H, Goodman PH, Berger TK, Ma J, Goldman-Rakic PS (2006) Heterogeneity in the pyramidal network of the medial prefrontal cortex. Nat Neurosci 9:534-542.

Wang Z, McCormick DA (1993) Control of firing mode of corticotectal and corticopontine layer $\mathrm{V}$ burst-generating neurons by norepinephrine, acetylcholine, and 1S,3R-ACPD. J Neurosci 13:2199-2216.

Wilson CJ (1987) Morphology and synaptic connections of crossed corticostriatal neurons in the rat. J Comp Neurol 263:567-580.

Wilson CJ (2004) Basal ganglia. In: The synaptic organization of the brain, Ed 5 (Shepherd G, ed), pp 361-413. New York: Oxford UP.

Yoshimura Y, Dantzker JL, Callaway EM (2005) Excitatory cortical neurons form fine-scale functional networks. Nature 433:868-873. 